\title{
A Theoretical Study of Rate Coefficients for the O + NO Vibrational Relaxation
}

\author{
P. J. S. B. Caridade, ${ }^{\dagger}$ V. C. Mota, ${ }^{\dagger}$ J. R. Mohallem, ${ }^{\dagger}$ and A. J. C. Varandas $*{ }^{\star \dagger}$ \\ Departamento de Química, Universidade de Coimbra, 3004-535 Coimbra, Portugal, and Departamento de \\ Física, ICEx, Universidade Federal de Minas Gerais, P.O. Box 702, 30123-970 Belo Horizonte, MG, Brasil
}

Received: July 11, 2007; In Final Form: October 31, 2007

\begin{abstract}
Quasi-classical trajectories have been integrated to study the vibrational relaxation of the $\mathrm{O}+\mathrm{NO}(v)$ process as a function of the initial vibrational quantum number for $T=298 \mathrm{~K}, 1500 \mathrm{~K}$, and $3000 \mathrm{~K}$. Two reliable potential energy surfaces have been employed for the $A^{\prime}$ and $A^{\prime \prime}$ doublet states of $\mathrm{NO}_{2}$. The calculated vibrational relaxation rate constants show a nearly $v$-independent behavior at room temperature and a moderate increase with $v$ for higher temperatures. Although deviating significantly from the recommended values, good agreement with recent experimental results has been obtained. The importance of multi-quantum transitions is also analyzed.
\end{abstract}

\section{Introduction}

The cooling down mechanism in the lower terrestrial thermosphere is essentially due to the fundamental one-quantum vibration-band emission from nitric oxide near $5.3 \mu \mathrm{m}{ }^{1,2}$ The mechanism considers that radiative cooling is a result of the infrared emission

$$
\mathrm{NO}(v=1) \rightarrow \mathrm{NO}(v=0)+h v(5.3 \mu \mathrm{m})
$$

which is enhanced at the maximum of the solar cycle and high latitudes as a consequence of the increase of the NO profile due to auroral dosing. ${ }^{3}$ The vibrational excited NO is assumed to be formed via collisions with surrounding oxygen atoms

$$
\mathrm{O}+\mathrm{NO}(v=0) \rightarrow \mathrm{O}+\mathrm{NO}(v=1)
$$

with the knowledge of the rate coefficients for such an excitation process being therefore of key importance for the energy budget at such altitudes. Usually, the required rate constant is obtained by using the principle of detailed balance: $k^{v=0 \rightarrow 1} / k^{v=1 \rightarrow 0}=$ exp$\left(-\Delta E / k_{\mathrm{B}} T\right)$, where $\Delta E=1875 \mathrm{~cm}^{-1}$ is the energy difference between the $v=0$ and $v=1$ vibrational states of the NO molecule. Other sources of vibrationally excited NO are wellknown, ${ }^{4-7}$ namely, the reactions

$$
\begin{gathered}
\mathrm{N}\left({ }^{4} S\right)+\mathrm{O}_{2} \rightarrow \mathrm{NO}(v \leq 7)+\mathrm{O} \\
\mathrm{N}\left({ }^{2} D\right)+\mathrm{O}_{2} \rightarrow \mathrm{NO}(v \leq 18)+\mathrm{O}
\end{gathered}
$$

Product NO molecules with high degrees of vibrational content have been observed in various experiments. For example, the CIRRIS 1A mission observed $\mathrm{NO}(v \leq 2)$ fundamental band radiance in high-resolution earthlimb emission, ${ }^{8}$ and up to $v=5$ excitations under naturally occurring auroral. ${ }^{9}$ In turn, the EXCEDE III space-based artificial aurora experiment ${ }^{10}$ has shown nitric oxide in vibrational states up to $v=12$.

Despite its key role in kinetic models of the lower thermosphere, the vibrational energy transfer of NO via atomic oxygen collisions has been a subject of controversy. The first experi-

* Corresponding author. E-mail address: varandas@qtvs1.qui.uc.pt.

$\dagger$ Universidade de Coimbra.

$\doteqdot$ Universidade Federal de Minas Gerais. mental measurements are attributed to Fernando and Smith ${ }^{11}$ in 1979 using laser-induced infrared fluorescence techniques to study the $v=1 \rightarrow 0$ process. Lilenfeld ${ }^{12}$ also studied the title process, with the direct excitation of $\operatorname{NO}(v=1)$ being performed via a pulsed $\mathrm{CO}$ laser line to pump a near-resonant $\mathrm{NO}(v=0 \rightarrow 1)$ rotational transition. More recently, rate constants for $v=1-3$ have been reported ${ }^{13-15}$ using laserinduced fluorescence, with the value of the $v=1 \rightarrow 0$ process being found to be about 3 times smaller than the ones of Fernando and Smith ${ }^{11}$ and Lilenfeld. ${ }^{12}$ The implication of such differences on the impact on the temperature and density structure of the thermosphere has been studied by Sharma and Roble $^{16}$ using the global mean model. ${ }^{17}$ They found that such structures would become unrealistic when using the smaller rateconstant values.

On the theoretical side, two different methodological studies have been reported in the literature. One of them ${ }^{18,19}$ used the statistical adiabatic channel (SAC) model based on the groundstate potential energy surface of $\mathrm{NO}_{2}$. In turn, Duff and Sharma ${ }^{20}$ performed a classical trajectory study of the title process by considering the ${ }^{2} A^{\prime}$ and ${ }^{2} A^{\prime \prime}$ states of $\mathrm{NO}_{2}$. Both studies show important limitations, leading to inconclusive results. In this work, we have run quasiclassical trajectories (QCTs) on two realistic potential energy surfaces for both the ${ }^{2} A^{\prime}$ and ${ }^{2} A^{\prime \prime}$ states of $\mathrm{NO}_{2}$ in an attempt to highlight the dynamics of the $\mathrm{O}+\mathrm{NO}$ vibrational relaxation. In section 2 , we discuss briefly the potential energy surfaces employed in this work, while in section 3 the technical details of the QCT method are presented. Section 4 is devoted to the discussion of the present results, with the major conclusions being gathered in section 5 .

\section{Potential Energy Surfaces}

Neglecting the spin-orbit effect, the $\mathrm{O}\left({ }^{3} P\right)+\mathrm{NO}\left({ }^{2} \Pi\right)$ channel correlates with six potential energy surfaces $\left(2,4 A^{\prime}, 2,4 A^{\prime \prime}\right)$. Of such an ansatz, only the two lowest doublet states of $A^{\prime}$ and $A^{\prime \prime}$ symmetry show stable minima, a necessary requirement for an effective energy transfer process. In fact, preliminary calculations performed on the ${ }^{4} A^{\prime}$ potential energy surface have shown that no significant vibrational relaxation occurs for the temperatures considered in the present work. As a result of such an ineffective energy transfer, only the electronic doublet states will be considered in the current work. 
TABLE 1: Numerical Data of the QCT Calculations for the Electronic-State Specific and Electronic-State Total State-to-All Vibrational Relaxation for the $\mathrm{O}+\mathrm{NO}(v) \rightarrow \mathrm{O}+\mathrm{NO}\left(v^{\prime}\right)$ Reaction

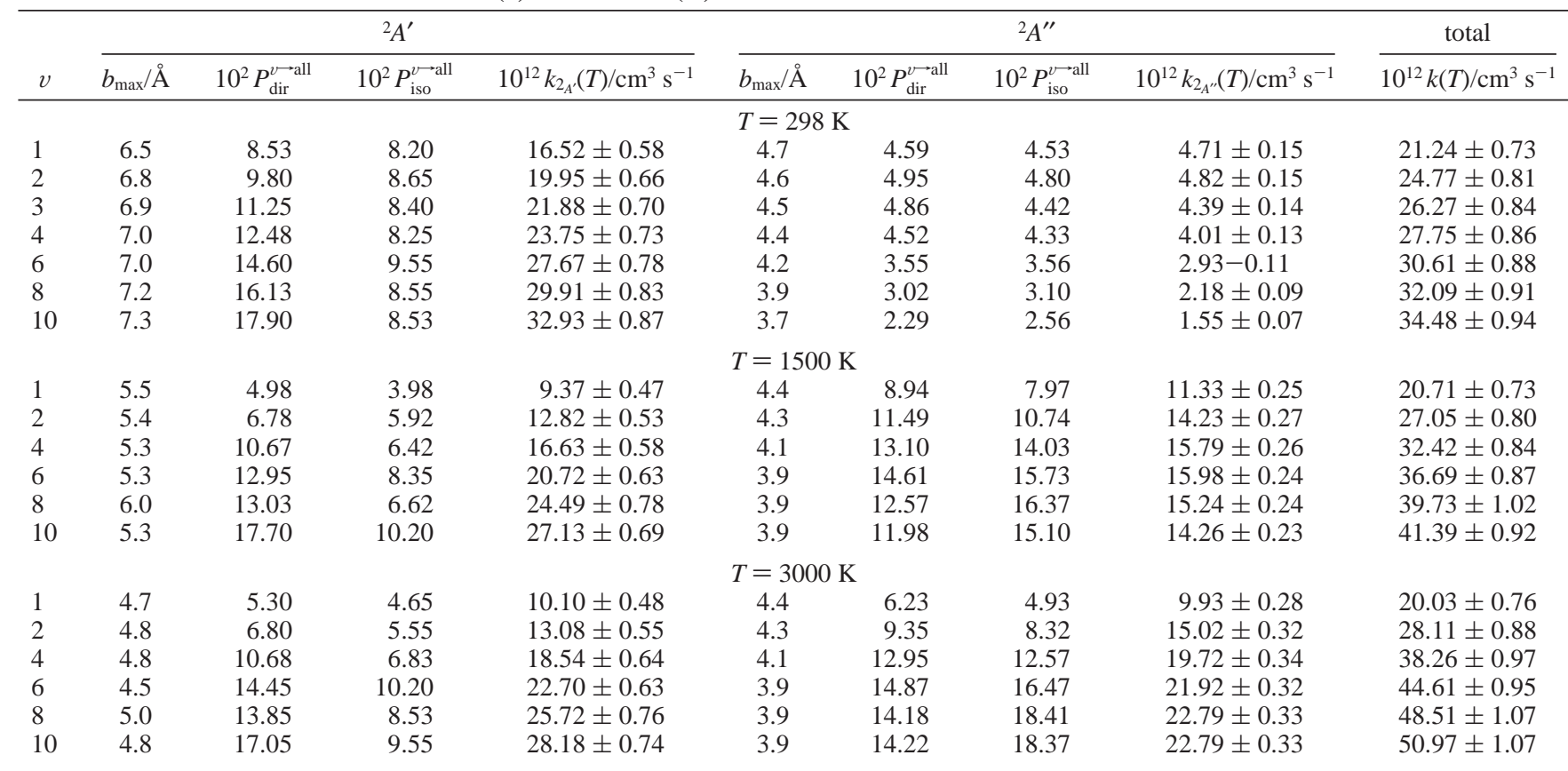

The ground ${ }^{2} A^{\prime}$ state of $\mathrm{NO}_{2}$ has been widely studied in the literature (ref 21, and references therein), with several potential energy surfaces having been reported for this system, focusing especially on the $\mathrm{N}\left({ }^{4} S\right)+\mathrm{O}_{2}$ reaction channel. In this study, we have employed the realistic multi-sheeted double many-body expansion $^{22}$ (DMBE) potential energy surface reported by Varandas, ${ }^{21}$ which has near spectroscopic accuracy in regions close to the minimum as achieved via a multiple energyswitching (ES) scheme. ${ }^{21,23}$ Using a minimal basis for $\mathrm{O}\left({ }^{3} P_{g}\right)$ and $\mathrm{N}\left({ }^{4} S_{u}\right)$, this DMBE potential energy surface is based on an $8 \times 8$ Hamiltonian that has been constructed using the diatomicin-molecules formalism (see, e.g., ref 24), with each matrix element then being corrected with three-body energy terms to account for the finite size of the basis sets as well as the missing many-center contributions and orbital overlaps. Such three-body correction terms, which have been included by dressing the input diatomic curves, have been calibrated using both theoretical and experimental data. After diagonalization, a manifold of eight ${ }^{2} A$ ' potential energy surfaces is obtained, with the lowest adiabatic state having been morphed with an effective spectroscopic polynomial form ${ }^{25}$ via a multiple $\mathrm{ES}^{21,23}$ scheme. The resulting adiabatic DMBE/ES potential energy surface mimics all the known attributes of the $\mathrm{NO}_{2}\left(1^{2} A^{\prime}\right)$ state, including an accurate description of the vibrational spectroscopy of groundstate $\mathrm{NO}_{2}$, and the $\mathrm{N}\left({ }^{4} S\right)+\mathrm{O}_{2}$ energy barrier for reaction. Also relevant is the accurate description of the crossings and avoided crossings that characterize this open-shell system. In addition, a study of the reaction $\mathrm{N}\left({ }^{4} S\right)+\mathrm{O}_{2}$ and its reverse on this DMBE/ ES potential energy surface has shown good agreement with experimental and other theoretical results. ${ }^{26}$

The number of reported studies for the $1^{2} A^{\prime \prime}$ electronic state is much smaller than that for the ground state. Gillespie et al. ${ }^{27}$ reported bending curves for the equilibrium NO distance of the $\mathrm{NO}_{2}$ molecule, which have been used by Duff and Sharma ${ }^{20}$ to construct a global potential energy surface. In turn, Braunstein and Duff ${ }^{28}$ have performed an ab initio study of the ${ }^{2} A^{\prime \prime}$ state using the complete active space self-consistent field (CASSCF) and second-order perturbation theory on a CASSCF wave function (CASPT2) methods with a $6311 \mathrm{G}(d)$ basis set. The reported energies were then used to construct a global many- body expansion ${ }^{29}$ (MBE), which shows a root-mean-squared deviation of $19.3 \mathrm{~kJ} \mathrm{~mol}^{-1}$. More recently, González et al. ${ }^{30}$ have also performed CASPT2 calculations but using a two-state averaged method and a more flexible cc-VTZ basis set. As in the previous case, the energies were used to calibrate a MBE function, with the fit showing a root-mean-square deviation of $2.47 \mathrm{kcal} \mathrm{mol}^{-1}$. Because of the more realistic nature of this potential energy surface in comparison to the one of Braunstein and Duff, ${ }^{28}$ we have employed the MBE function of ref 30 in our work. The two major topographical characteristics of this surface relevant for the present work are the absence of a barrier for the $\mathrm{O}\left({ }^{3} P\right)+\mathrm{NO}$ reaction, and the fact that the absolute minimum shows $C_{2 v}$ symmetry. It should also be noted that this potential energy surface has been used to study the $\mathrm{N}\left({ }^{2} D\right)$ $+\mathrm{O}_{2}$ reaction, with the results showing good agreement with experiment. ${ }^{30}$

\section{QCT Calculations}

The dynamics study reported in this work utilizes the QCT method, which is well described in the literature. ${ }^{31}$ For an initial vibrational state $v$, the total relaxation rate constant assumes the form

$$
k^{v \hookrightarrow \text { all }}(T ; v)=k_{2_{A^{\prime}}}^{v \rightarrow \text { all }}(T ; v)+k_{2_{A^{\prime}}^{\prime}}^{v \rightarrow \text { all }}(T ; v)
$$

Since the two potential energy surfaces here employed have different symmetries, the calculations are carried out separately. Assuming a Boltzmann distribution for the translational and rotational energies, the rate constant for state-to-all vibrational relaxation is given by

$$
k_{x}^{v \rightarrow \text { all }}(T ; v)=g_{\mathrm{e}}(T)\left(\frac{8 k_{\mathrm{B}} T}{\pi \mu_{\mathrm{O}+\mathrm{NO}}}\right)^{1 / 2} \sum_{j} \frac{(2 j+1) \exp \left(-E_{j ; v} / k_{\mathrm{B}} T\right)}{Q_{j ; v}} \times
$$

where $g_{\mathrm{e}}(T)=2 /\{[5+3 \exp (-227.8 / T)+\exp (-326.6 / T)](2$ $+2 \exp (-177.1 / T))\}$ is the electronic degeneracy factor, $k_{\mathrm{B}}$ is the Boltzmann constant, $E_{j ; v}$ is the rovibrational energy for the 

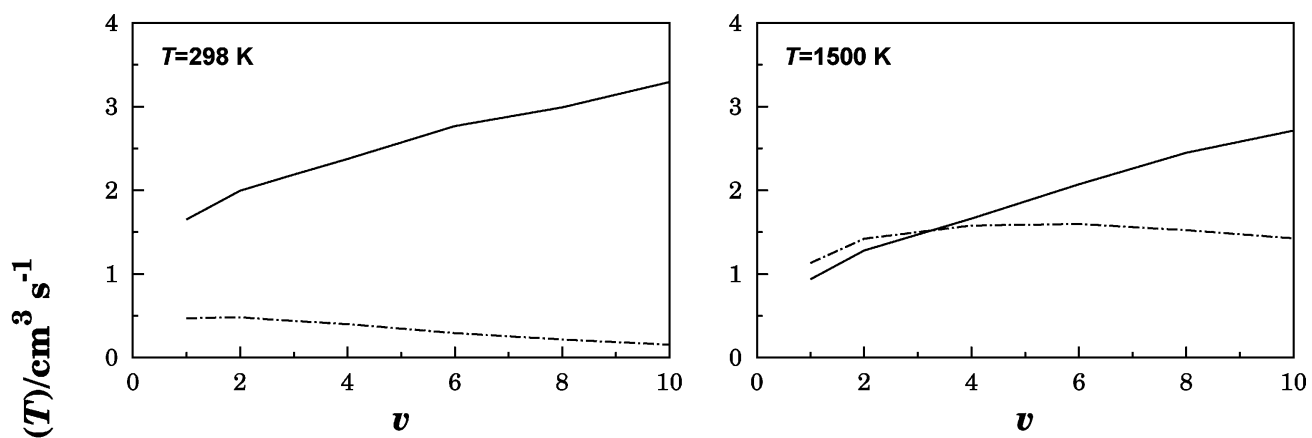

\section{$\mathrm{O}+\mathrm{NO}(v) \rightarrow \mathrm{NO}($ all $)+\mathrm{O}$}

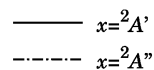

Figure 1. Electronic-sta considered in this work.

$(v, j)$ state, $Q_{j ; v}$ is the rotational partition function for the $v$ state, $\mu_{\mathrm{O}+\mathrm{NO}}$ is the reduced mass of the reactants, and $\sigma\left(E_{t r} ; j v\right)$ is the vibrational-specific relaxation cross section. To calculate the integral in eq 6, we have used a random sampling of $E_{\mathrm{tr}}$ from a Maxwell-Boltzmann distribution via the cumulative distribution function ${ }^{31,32}$

$$
E_{\mathrm{tr}}=-k_{\mathrm{B}} T \ln \left(\xi_{1} \xi_{2}\right)
$$

where $\xi_{1}$ and $\xi_{2}$ are two independent uniform random numbers (for an alternative translational energy sampling, see ref 33 and references therein).

For each vibrational quantum number and trajectory, the rotational quantum number is chosen from a Boltzmann distribution using the cumulative function ${ }^{34}$

$$
\sum_{j_{0}}^{j} Q_{j ; v}^{-1}(2 j+1) \exp \left(-E_{j ; v} / k_{\mathrm{B}} T\right) \geq \xi_{3}
$$

with the summation running until the condition is verified for each freshly generated random number $\xi_{3}$. As in previous studies, ${ }^{26} E_{j ; v}$ is calculated from the numerical solution of the Schrödinger equation for the $\mathrm{NO}\left({ }^{2} \Pi\right)$ curves used to describe the diatomic fragments of both potential energy surfaces employed in this work. Since NO is a ${ }^{2} \Pi$ state, the minimum rotational quantum number is $j_{0}=1$. All the remaining sampling parameters are chosen by the standard procedure. ${ }^{32}$

Batches of $N=4000$ and 10000 trajectories have been performed on the DMBE/ES and MBE potential energy surfaces, respectively, for $v=1-10$ and $T=298,1500$ and $3000 \mathrm{~K}$, with a time step of $2 \times 10^{-17} \mathrm{~s}$. Such a number of trajectories is sufficient to minimize fluctuations in the rate constant, which should be converged to within $1 \%$. The optimization of the impact parameter has been carried out assuming that the last bin should be empty for the vibrational relaxation process. In turn, the calculation of the final vibrational state has been carried out by using the semi-classical quantization method ${ }^{32}$ and rounding the real value to the nearest integer. By replacing the above sampling procedures in eq 6 , one gets for each potential energy surface

$$
k_{x}^{v \rightarrow \text { all }}(T ; v)=g_{\mathrm{e}}(T)\left(\frac{8 k_{\mathrm{B}} T}{\pi \mu_{\mathrm{O}+\mathrm{NO}}}\right)^{1 / 2} \pi b_{\max }{ }^{2} P^{v \rightarrow \text { all }}
$$

where $P^{v \rightarrow \text { all }}=N^{v \rightarrow \text { all }} / N$, and $\Delta k_{x}^{v \rightarrow \text { all }}(T ; v)=k_{x}^{v \rightarrow \text { all }}(T ; v)[(N-$ $\left.\left.N^{v \rightarrow \text { all }}\right) / N N^{v \rightarrow \text { all }}\right]^{1 / 2}$. A similar expression can be obtained for the state-to-state rate constant by replacing $N^{v \rightarrow \text { all }}$ by $N^{v \rightarrow v^{\prime}}$. A final remark to note that both the direct vibrational relaxation

$$
\mathrm{O}_{\mathrm{a}}+\mathrm{NO}_{\mathrm{b}}(v) \rightarrow \mathrm{O}_{\mathrm{a}}+\mathrm{NO}_{\mathrm{b}}\left(v^{\prime}\right)
$$

and the relaxation via exchange reaction

$$
\mathrm{O}_{\mathrm{a}}+\mathrm{NO}_{\mathrm{b}}(v) \rightarrow \mathrm{O}_{\mathrm{b}}+\mathrm{NO}_{\mathrm{a}}\left(v^{\prime}\right)
$$

are counted for the total vibrational relaxation rate constants.

\section{Results and Discussion}

Table 1 and Figure 1 gather the electronic-state specific stateto-all vibrational relaxation rate constants calculated using the ${ }^{2} A$ and ${ }^{2} A$ " potential energy surfaces. Depending on the temperature, one observes different patterns. For room-temperature, the dominant contribution comes from the rate constant for the ${ }^{2} A^{\prime}$ potential energy surface, which is 3 to 7 times larger than the ${ }^{2} A$ " one. Such behavior has also been found in the QCT study by Duff and Sharma, ${ }^{20}$ although their results show a nearly $v$-independent behavior and a smaller scaling ratio $(25 \%)$. For the other temperatures here examined, both of the above contributions become of similar value for lower vibrational states, while for higher ones the ${ }^{2} A^{\prime}$ dominance becomes attenuated with increasing temperature. The distinct dynamical attributes of the ${ }^{2} A^{\prime}$ and ${ }^{2} A^{\prime \prime}$ potential energy surfaces can be rationalized from their different topographical features. The most important feature distinguishing the two surfaces refers to the well depth of the $C_{2 v}$ minimum: $311.7 \mathrm{~kJ} \mathrm{~mol}^{-1}$ for the ${ }^{2} A^{\prime}$ state $^{21}$ and $56.9 \mathrm{~kJ} \mathrm{~mol}^{-1}$ for ${ }^{2} A^{\prime \prime} 30$ (relative to the $\mathrm{O}+\mathrm{NO}$ asymptote). As expected, the deeper minimum of the ${ }^{2} A^{\prime}$ state 


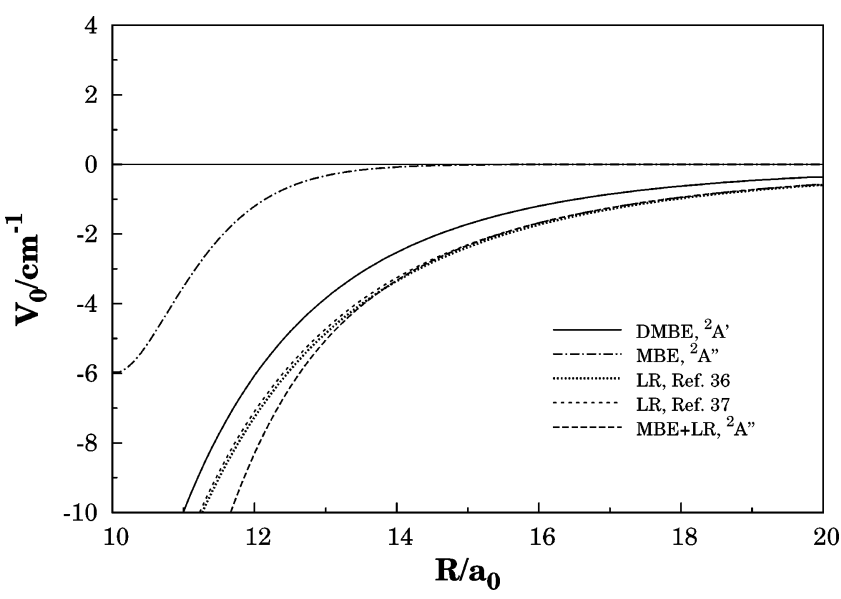

Figure 2. Spherically averaged component of the $\mathrm{O}\left({ }^{3} P\right)-\mathrm{NO}\left({ }^{2} \Pi\right)$ interaction for the DMBE/ES, MBE, and MBE+LR potential energy surfaces. Also shown for comparison are the long-range potentials from refs 36 and 37.

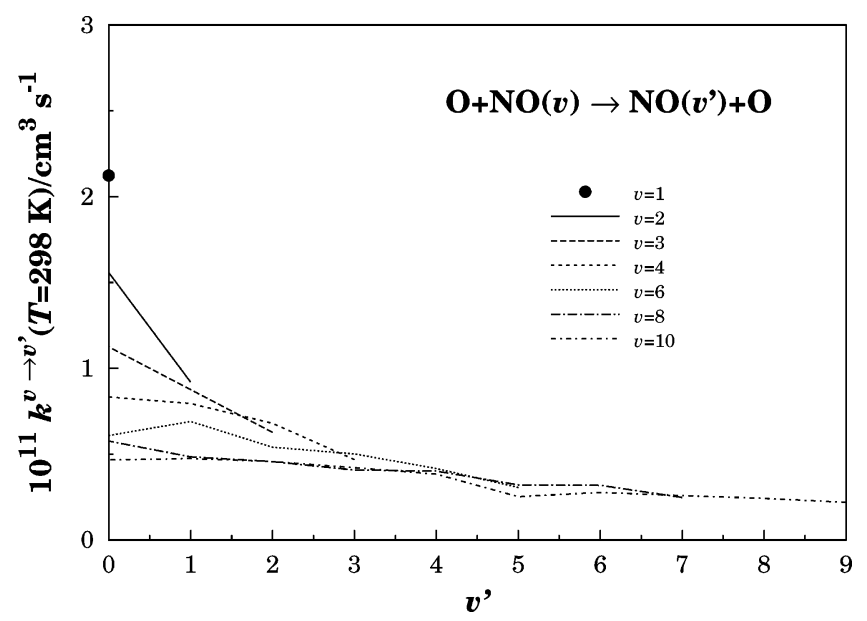

Figure 3. Thermal state-to-state rate constants for $T=298 \mathrm{~K}$.

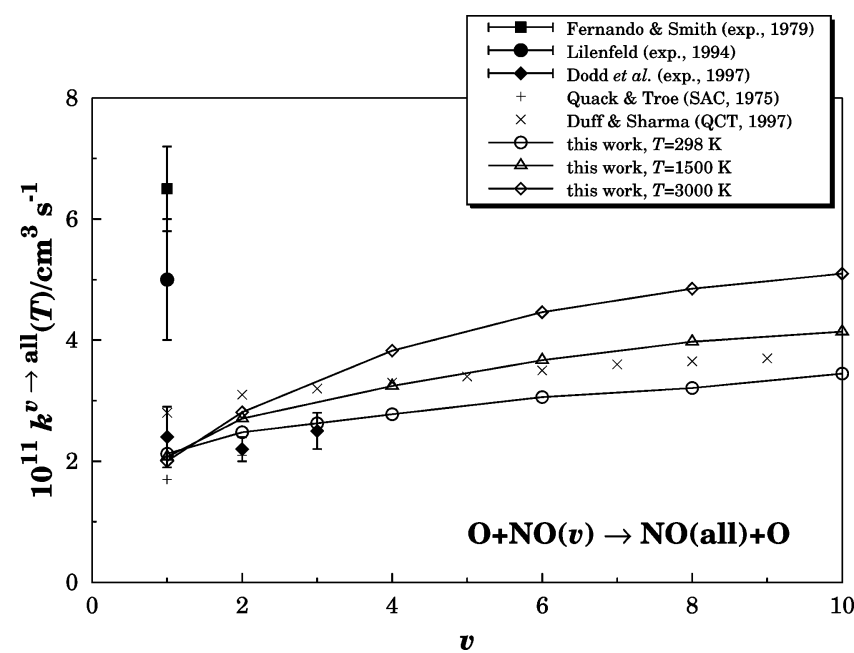

Figure 4. Thermal state-to-all rate constants as a function of the initial vibrational quantum number for the three different temperatures considered in this work. Also shown are experimental ${ }^{11,12,14,15}$ and theoretical ${ }^{19,20}$ data found in the literature.

should allow a more efficient randomization of the energy in the vibrational modes of $\mathrm{NO}_{2}$, and hence a larger probability of energy transfer. As the temperature raises, the number of trajectories that sample regions near the potential minimum decreases and the differences in the ${ }^{2} A^{\prime}$ and ${ }^{2} A^{\prime \prime}$ rate constants become less significant.
An important issue on the dynamics of the title collisional process refers to the role of long-range forces, ${ }^{11}$ a subject that has also been addressed in the study of $\mathrm{NO}_{2}$ recombination in the high-pressure limit. ${ }^{35,36}$ Similarities arise because both processes are capture-type, occurring in a barrier-free potential energy surface with a potential well. The DMBE/ES ${ }^{21}$ form is expected to have an approximate long-range behavior due to the use of realistic two-body energy curves. This is corroborated from Figure 2, which shows its leading spherically averaged term for the $\mathrm{O}-\mathrm{NO}$ interaction as obtained with NO fixed at its diatomic equilibrium geometry. For comparison, we also show the corresponding long-range terms from the multipolar expansions of Harding et al. ${ }^{36}$ and Reignier et al. ${ }^{37}$ Although based on the same long-range coefficients, the former differs from the latter only because this includes higher-order dispersion interaction coefficients $\left(C_{8}\right.$ and $\left.C_{10}\right)$, with all long-range coefficients also carrying damping functions that account for charge-overlap and exchange effects. ${ }^{38}$ Note that the dominant contribution for the interaction between $\mathrm{O}\left({ }^{3} P\right)$ and $\mathrm{NO}\left({ }^{2} \Pi\right)$ arises from the electrostatic term involving the quadrupole moment of the oxygen atom and the dipole moment of NO, $C_{4}$. To represent such electrostatic terms, we have utilized a variant of the optimized quadrupole-moment scheme (ref 39, and references therein) whereby the orientation angle of the atomic quadrupole is chosen to be fixed at the value that maximizes the attraction of the atom-diatom electrostatic interaction (cos $\theta=1$ ). Also seen from Figure 2 is the fairly good agreement (deviations do not exceed $1 \mathrm{~cm}^{-1}$ or so for the separations shown in Figure 2) between the long-range interaction arising from the DMBE/ES potential energy surface and the above-mentioned spherically average expansions.

As a result of construction, the MBE potential for the ${ }^{2} A^{\prime \prime}$ electronic state does not show the appropriate long-range behavior (see Figure 2). To correct it, we have added a term that approximately mimics the desired behavior. The approach consists of adding to the original MBE form the electrostatic and dispersion terms taken from ref 37 suitably damped as described in the previous paragraph. Cuts of the potential energy surface so obtained have been examined to make sure that no spurious attributes were present. Figure 2 shows the spherically averaged component of the final modified potential energy surface $(\mathrm{MBE}+\mathrm{LR})$. As seen, the numerically integrated $V_{0}$ from MBE+LR coincides, as it should, with the long-range one over the distances relevant for the capture process. It has therefore been employed for test dynamics studies at $T=298 \mathrm{~K}$. The vibrational relaxation rate constant calculated from it for the $v$ $=1 \rightarrow 0$ process is $k_{2_{A^{\prime \prime}}}(T=298 \mathrm{~K})=9.46 \pm 0.20 \mathrm{~cm}^{-3} \mathrm{~s}^{-1}$, thus a factor of 2 or so larger than the one obtained with the original MBE function. Despite this twofold increase, the dominant contribution still arises from the ${ }^{2} A^{\prime}$ potential energy surface, and hence the new rate constant will not significantly alter the final conclusions prior to using MBE+LR (i.e., obtained with the unmodified MBE form for the upper excited state).

Table 2 gathers the state-to-state vibrational relaxation rate constants and branching ratios, while Figure 3 illustrates them for $T=298 \mathrm{~K}$. A salient feature is the importance of multiquanta transitions, which is also observed for higher temperatures. Since vibrational relaxation is expected to occur in surfaces dominated by strongly bound complexes, this may explain the observed high probability for vibrational multiquanta transitions and also the fact that the $v^{\prime}=0$ state is the most populated product state. Such a finding has been previously reported by Duff and Sharma $^{20}$ who noted that "the SAC model for $\mathrm{NO}(v \leq 1,2)$ and the expectations of statistical theories predict that $v^{\prime}=0$ 
TABLE 2: State-to-State Vibrational Relaxation Rate Constants and Branching Ratios for the $\mathrm{O}+\mathrm{NO}(v) \rightarrow \mathrm{O}+\mathrm{NO}\left(v^{\prime}\right)$ Reaction

\begin{tabular}{|c|c|c|c|c|c|c|c|c|c|c|}
\hline \multirow[b]{2}{*}{$v$} & \multicolumn{10}{|c|}{$10^{12} k^{v \diamond v^{\prime}}\left(\chi_{v \diamond v^{\prime}}\right)$} \\
\hline & $v^{\prime}=0$ & 1 & 2 & 3 & 4 & 5 & 6 & 7 & 8 & 9 \\
\hline \multicolumn{11}{|c|}{$T=298 \mathrm{~K}$} \\
\hline 1 & $21.24(1.000)$ & & & & & & & & & \\
\hline 2 & $15.58(0.629)$ & $9.20(0.371)$ & & & & & & & & \\
\hline 3 & $11.25(0.428)$ & $8.76(0.333)$ & $6.26(0.238)$ & & & & & & & \\
\hline 4 & $8.33(0.300)$ & $7.95(0.286)$ & $6.79(0.245)$ & $4.68(0.169)$ & & & & & & \\
\hline 6 & $6.08(0.199)$ & $6.90(0.226)$ & $5.41(0.177)$ & $5.01(0.164)$ & $4.16(0.136)$ & $3.05(0.100)$ & & & & \\
\hline 8 & $5.76(0.179)$ & $4.84(0.151)$ & $4.56(0.142)$ & $4.07(0.127)$ & $4.01(0.125)$ & $3.20(0.100)$ & 3.18 (0.099) & $2.47(0.077)$ & & \\
\hline 10 & $4.66(0.135)$ & $4.74(0.138)$ & $4.57(0.133)$ & $4.21(0.122)$ & $3.83(0.111)$ & $2.52(0.073)$ & $2.77(0.080)$ & $2.58(0.075)$ & $2.42(0.070)$ & $2.19(0.063)$ \\
\hline \multicolumn{11}{|c|}{$T=1500 \mathrm{~K}$} \\
\hline 1 & $20.71(1.000)$ & & & & & & & & & \\
\hline 2 & $15.14(0.559)$ & $11.91(0.440)$ & & & & & & & & \\
\hline 4 & $8.98(0.277)$ & $8.95(0.276)$ & $7.53(0.232)$ & $6.96(0.214)$ & & & & & & \\
\hline 6 & $6.98(0.190)$ & $7.31(0.199)$ & $6.16(0.168)$ & $6.13(0.167)$ & $4.97(0.135)$ & $5.14(0.140)$ & & & & \\
\hline 8 & $6.30(0.158)$ & $5.71(0.14)$ & $5.84(0.147)$ & $5.04(0.127)$ & $4.75(0.120)$ & $4.59(0.116)$ & $3.89(0.098)$ & $3.62(0.091)$ & & \\
\hline 10 & $5.48(0.132)$ & $5.25(0.127)$ & $4.94(0.119)$ & $4.48(0.108)$ & $4.27(0.103)$ & $3.76(0.091)$ & $3.73(0.090)$ & $3.62(0.087)$ & $3.06(0.074)$ & $2.82(0.068)$ \\
\hline \multicolumn{11}{|c|}{$T=3000 \mathrm{~K}$} \\
\hline 1 & $20.03(1.000)$ & & & & & & & & & \\
\hline 2 & $14.57(0.518)$ & $13.53(0.482)$ & & & & & & & & \\
\hline 4 & $10.38(0.271)$ & $10.09(0.264)$ & $9.33(0.244)$ & $8.46(0.221)$ & & & & & & \\
\hline 6 & $8.19(0.184)$ & $8.43(0.189)$ & $7.46(0.167)$ & 7.10 (0.159) & $6.33(0.142)$ & $7.10(0.159)$ & & & & \\
\hline 8 & $7.19(0.148)$ & $6.32(0.130)$ & $6.63(0.137)$ & $6.44(0.133)$ & $6.03(0.124)$ & $5.51(0.114)$ & $4.70(0.097)$ & $5.68(0.117)$ & & \\
\hline 10 & $5.85(0.115)$ & $5.98(0.117)$ & $5.26(0.103)$ & $6.24(0.122)$ & $4.90(0.096)$ & $4.94(0.097)$ & $4.58(0.090)$ & $4.34(0.085)$ & $4.28(0.084)$ & $4.62(0.091)$ \\
\hline
\end{tabular}

should be the most populated final vibrational state independent of initial $v$ ". Despite the above observation, the mechanism of the title reaction cannot be considered as purely statistical. In fact, Table 1 suggests that the direct vibrational relaxation channel in ${ }^{2} A^{\prime}$ state dominates over chemical relaxation. In a pure statistical event and in absence of steric effects that control chemical reaction, one might expect energy randomization to take place with an equal probability by inelastic energy transfer and reactive deactivation, as appears to be the case for collisions evolving in the ${ }^{2} A^{\prime \prime}$ state. Regarding the branching ratios, the experimental measurements ${ }^{14}$ indicate a value of $\chi(v=3 \rightarrow 2)$ $=0.35 \pm 0.12$. Our theoretical estimate of $\chi(v=3 \rightarrow 2)=$ 0.24 shows a fair agreement (see Table 2), which becomes good when comparing with the result $(0.29 \pm 0.02)$ from other QCT calculations. ${ }^{20}$

Figure 4 and the last column of Table 1 show the total stateto-all vibrational relaxation rate constants as a function of the initial $v$ state for the three temperatures studied in the current work. As expected from the previous paragraphs, the rate constant for room-temperature shows a slight increase due to partial cancellation of the two state contributions. For higher temperatures, the most significant increase occurs for high initial vibrational states, mainly due to the ${ }^{2} A$ " contribution (see Figure 1 ), while for $v=1$ and 2 the rate constant is nearly temperatureindependent. For comparison, we also show in Figure 4 other theoretical data from the literature. The QCT values of ref 20 run parallel to ours, albeit a factor of nearly 2 larger, especially for low vibrational states. This can be attributed to a somewhat cruder modeling of their potential energy surface. Recall that the global potential energy surfaces used in their work were calibrated from ab initio energies for a single $C_{2 v}$ bending cut originated from Hirsch and Buenker ${ }^{40}$ for the ${ }^{2} A^{\prime}$ state, and Gillispie et al. ${ }^{27}$ for the ${ }^{2} A^{\prime \prime}$ one. Quack and Troe ${ }^{19}$ calculated the vibrational relaxation rate constants using the SAC model based only the ${ }^{2} A^{\prime}$ topographical attributes. Their reported values of $k^{v=1 \rightarrow 0}(T=300 \mathrm{~K})=1.7 \times 10^{-11} \mathrm{~cm}^{3} \mathrm{~s}^{-1}$ and $k^{v=1 \rightarrow \text { all }}(T=$ $300 \mathrm{~K})=2.1 \times 10^{-11} \mathrm{~cm}^{3} \mathrm{~s}^{-1}$ are approximately $20 \%$ smaller than our results. Such differences can be attributed to having considered only the ${ }^{2} A^{\prime}$ state in the SAC model. In fact, if one considers the Quack-Troe ${ }^{19}$ estimates and adds the ${ }^{2} A^{\prime \prime}$ contribution calculated in this work, the agreement with the QCT results is strikingly good: $k^{v=1 \rightarrow 0}=2.2 \times 10^{-11} \mathrm{~cm}^{3}$ and $k^{v=2 \rightarrow \text { all }}$ $=2.6 \times 10^{-11} \mathrm{~cm}^{3} \mathrm{~s}^{-1}$ for the SAC versus 2.1 and $2.5 \times 10^{-11}$ $\mathrm{cm}^{3} \mathrm{~s}^{-1}$ for QCT, respectively.

Also shown for comparison in Figure 4 are the available experimental data for the title system. Clearly, the $k^{v=1 \rightarrow 0}$ for room temperature shows very good agreement with the reported value of Dodd et al., ${ }^{15}$ but deviates somewhat from the recommended values of Fernando and Smith ${ }^{11}$ and Lilenfeld. ${ }^{12}$ A good agreement ${ }^{14}$ is also observed for the vibrational states $v=2$ and 3. Although not shown in Figure 4, Glazer and Troe ${ }^{18}$ have studied the vibrational relaxation of $\operatorname{NO}(v=1,2)$ by shock-tube experiments for $T=2700 \mathrm{~K}$, having reported $k^{v=1 \rightarrow 0}$ $=3.65 \pm 1.66 \times 10^{-11} \mathrm{~cm}^{3} \mathrm{~s}^{-1}$ and $k^{v=2 \rightarrow \text { all }}=3.99 \pm 1.66 \times$ $10^{-11} \mathrm{~cm}^{3} \mathrm{~s}^{-1}$. Our QCT result for $T=3000 \mathrm{~K}$ is a factor of nearly 2 smaller, but falls almost within the experimental error bar. As discussed above, the inclusion of long-range forces on the MBE form for the ${ }^{2} A^{\prime \prime}$ state should not significantly alter the calculated vibrational relaxation rate constant. In fact, the corrected value for the $v=1 \rightarrow 0$ transition, is now predicted to be $k(T=298 \mathrm{~K})=2.59 \pm 0.58 \times 10^{-11} \mathrm{~cm}^{-3} \mathrm{~s}^{-1}$, in even closer agreement with the Dodd et al. ${ }^{15}$ experimental value, and still far from the recommended ${ }^{11}$ one.

\section{Conclusions}

In this work we have reported a QCT study of the $\mathrm{O}+\mathrm{NO}$ $(v)$ vibrational relaxation process as a function of the initial vibrational quantum number for three temperatures. Two realistic potential energy surfaces for the $A^{\prime}$ and $A^{\prime \prime}$ doublet states of $\mathrm{NO}_{2}$ have been utilized. The results show that, for room temperature, the dominant contribution arises from the ${ }^{2} A^{\prime}$ state, while for higher temperatures both states contribute almost equally. The state-to-state rate coefficients and branching ratios are predicted to be nearly independent of the final vibrational state, which may suggest a strong statistical behavior of the title energy transfer process. Calculated state-to-all rate constants for room temperature were also found to be in good agreement with the reported experimental values, ${ }^{13-15}$ albeit a factor of nearly 3 or so smaller than the recommended values of Fernando 
and Smith. ${ }^{11}$ Such data may bear important implications on the thermospheric temperature modeling as referred to in ref 3.

Acknowledgment. This work has been carried out under the auspices of Fundação para a Ciência e a Tecnologia, Portugal (contracts POCI/QUI/60501/2004, POCI/AMB/60261/2004, and REEQ/128/QUI/2005). The support from the bilateral GRICESCAPES program is also gratefully acknowledged.

\section{References and Notes}

(1) Kockarts, G. Geophys. Res. Lett. 1980, 7, 137.

(2) Zachor, A.; Sharma, R. D.; Nadile, R. M.; Stair, A. T., Jr. J. Geophys. Res. 1985, 90, 9776.

(3) Sharma, R. D.; Roble, R. G. ChemPhysChem 2002, 3, 841. 11331

(4) Rusch, D. W.; Gerard, J.; Fesen, C. G. J. Geophys. Res. 1991, 96,

(5) Barth, C. A. Planet. Space Sci. 1992, 40, 315

(6) Sharma, R. D.; Kharchenko, V. A.; Sun, Y.; Dalgarno, A. J. Geophys. Res. 1996, 101, 275.

(7) Gerard, J.; Bisikalo, D. V.; Shematovich, V. I.; Duff, J. W. J. Geophys. Res. 1997, 102, 285.

(8) Sharma, R. D.; von Esse, H. D. F.; Kharchenko, V. A.; Sun, Y.; Dalgarno, A. J. Geophys. Res. 1996, 101, 19707.

(9) Armstrong, P. S.; Lipson, S. J.; Dodd, J. A.; Lowell, J. R.; Blumberg, W. A. M.; Nadile, R. M. Geophys. Res. Lett. 1994, 21, 2425

(10) Armstrong, P. S.; Dodd, J. A.; Lipson, S. J.; Blumberg, W. A. M.; Lockwood, R. B.; Lowell, J. R.; Nadile, R. M.; Paulsen, D. E.; Murphy, R. E.; Rawlins, W. T.; Fraser, M. E.; Green, B. D. Eos Trans. AGU 1995, 76 , F439.

(11) Fernando, R. P.; Smith, I. W. M. Chem. Phys. Lett. 1979, 66, 218.

(12) Lilenfeld, H. V. Phillips Laboratory Final Report PL-T R-94-2180; Phillips Laboratory: Kirtland Air Force Base, NM, 1994.

(13) Dodd, J. A.; Singleton, S. M.; Miller, S. M.; Armstrong, P. S.; Blumberg, W. A. M. Chem. Phys. Lett. 1996, 260, 103.

(14) Dodd, J. A.; Lockwood, R. B.; Miller, S. M.; Blumberg, W. A. M. J. Chem. Soc., Faraday Trans. 1997, 93, 2637.

(15) Dodd, J. A.; Lockwood, R. B.; Hwang, E. S.; Miller, S. M. J. Chem Phys. 1999, 111, 3498

(16) Sharma, R. D.; Roble, R. G. J. Geophys. Res. 2001, 106, 21343.
(17) Roble, R. G. In The Upper Mesosphere and Lower Thermosphere A Review of Experiment and Theory; Johnson, R. M., Killeen, T. L., Eds.; American Geophysical Union: Washington, DC, 1995; p 1.

(18) Glanzer, K.; Troe, J. J. Chem. Phys. 1975, 63, 4352.

(19) Quack, M.; Troe, J. Ber. Bunsen-Ges. Phys. Chem. 1975, 79, 170.

(20) Duff, J. W.; Sharma, R. D. J. Chem. Soc., Faraday Trans. 1997, 93, 2645.

(21) Varandas, A. J. C. J. Chem. Phys. 2003, 119, 2596.

(22) Varandas, A. J. C. Advanced Series in Physical Chemistry; Yarkony, D. R., Köppel, H., Domcke, W., Eds.; World Scientific Publishing: River Edge, NJ, 2004; Chapter 5, p 91.

(23) Varandas, A. J. C. J. Chem. Phys. 1996, 105, 3524

(24) Kuntz, P. J. In Atom-Molecule Collision Theory; Bernstein, R., Ed.; Plenum: New York, 1979; p 79.

(25) Schryber, J. H.; Polyansky, O. L.; Jensen, P.; Tennyson, J. J. Mol. Spectrosc. 1997, 185, 234

(26) Caridade, P. J. S. B.; Varandas, A. J. C. J. Phys. Chem. A 2004 108,3556 .

(27) Gillespie, G. D.; Khan, A. U.; Wahl, A. C.; Hosteny, R. P.; Krauss, M. J. Chem. Phys. 1975, 63, 3425.

(28) Braunstein, M.; Duff, J. W. J. Chem. Phys. 2000, 113, 7406

(29) Murrell, J. N.; Carter, S.; Farantos, S. C.; Huxley, P.; Varandas, A. J. C. Molecular Potential Energy Functions; Wiley: Chichester, U.K., 1984

(30) González, M.; Miquel, I.; Sayós, R. J. Chem. Phys. 2001, 115, 8838.

(31) Peslherbe, G. H.; Wang, H.; Hase, W. L. Adv. Chem. Phys. 1999, 105,171

(32) Hase, W. L.; Duchovic, R. J.; Hu, X.; Komornicki, A.; Lim, K. F.; Lu, D.; Peslherbe, G. H.; Swamy, K. N.; Linde, S. R. V.; Varandas, A. J. C.; Wang, H.; Wolf, R. J. OCPE Bull. 1996, 16, 43

(33) Varandas, A. J. C.; Brandão, J.; Pastrana, M. R. J. Chem. Phys. 1992, 96, 5137.

(34) Rodrigues, S. P. J.; Varandas, A. J. C. J. Phys. Chem. A 2003 107,5369

(35) Hippler, H.; Siefke, M.; Stark, H.; Troe, J. Phys. Chem. Chem. Phys. 1999, 1, 57.

(36) Harding, L. B.; Stark, H.; Troe, J.; Ushakov, V. G. Phys. Chem. Chem. Phys. 1999, 1, 63

(37) Reignier, D.; Stoecklin, T.; Voronin, A.; Rayez, J. C. Phys. Chem Chem. Phys. 2001, 3, 2726.

(38) Varandas, A. J. C. J. Mol. Struct. (THEOCHEM) 1985, 120, 401.

(39) Varandas, A. J. C. J. Mol. Struct. (THEOCHEM) 1988, 166, 59.

(40) Hirsch, G.; Buenker, R. J. Can. J. Chem. 1985, 63, 1542. 